\title{
Intensive care of the surgical patient
}

\author{
L. J. LAWS ON \\ North Staffordshire Royal Infirmary
}

THE value of treatment in an Intensive Care Unit for patients undergoing cardiac or pulmonary surgery is well established. Few forms of general surgery require such treatment since it is exceptional to find any great change in body physiology during or after operation. Surgical procedures which benefit from intensive patient care may be divided into three categories.

\section{Elective admission to an Intensive Care Unit after extensive surgery}

Vascular surgery

Resection of aortic aneurysm.

Renal artery surgery.

Surgery of great vessels of the neck.

Mesenteric arterioplasty.

\section{Major replacement surgery}

Total oesophagectomy or pharyngo-laryngectomy with colon graft.

\section{Endocrine surgery}

Surgery of the adrenal gland.

Phaeochromocytoma.

Cushing's Disease.

\section{Elective admission to the Intensive Care Unit of poor risk cases}

This group includes patients with such conditions as coronary artery disease, respiratory insufficiency or severe diabetes mellitus. In such patients the operative procedure itself may not be formidable; partial gastrectomy, colectomy or emergency surgery for haemorrhage, perforation or intestinal obstruction, for example, often present no great technical problems, but post-operative care of the cardiorespiratory system may be very difficult.

\section{Emergency admission to the Intensive Care Unit}

These cases are encountered when cardiac arrest or respiratory insufficiency develops during or after uneventful surgery.

During a 12-month period, out of 442 admissions to the Intensive Care Unit, at the Department of Surgery, Queen Elizabeth Hospital, 128 cases fell into the above categories. Forty patients were admitted following gastro-intestinal surgery and the remainder had undergone some form of vascular surgery. In addition, twenty-six cases were admitted because cardiac or respiratory complications had followed relatively minor procedures. During this period the total number of beds in use at any one time was six, and the mean in-patient duration was $2 \cdot 4$ days.

The demands placed by such a wide variety of cases vary considerably. Patients admitted following aortic aneurysmectomy, for example, in whom complications do not occur, merely require routine recording of blood pressure, urine output and supervision of intravenous therapy. Those patients admitted with respiratory insufficiency, on the other hand, require ventilation, frequent estimates of $\mathrm{pH}$, $\mathrm{PCO}_{2}$, etc., as well as full nursing care.

Complete recording of information is essential for the correct functioning of an Intensive Care Unit. Electronic equipment for measuring heart rate and blood pressure is available, with suitable warning devices to give notice of emergency situations. It is advantageous to use fully automated equipment, combined with direct recording, but in our Unit data were measured and recorded by members of the nursing staff. Close observation of all the measured parameters permits the general condition of the patient to be assessed rapidly and the influence of specific therapy identified. Alteration of a parameter in a specific direction is particularly helpful since appropriate action can be planned before emergency treatment is needed. A computer system would facilitate this service, but as yet there is no functioning physiological system available using a computer mechanism. Considerable advantage could be derived from such equipment, since it would be possible to demonstrate 'trends', as well as producing a considerable saving in nursing time.

All surgical patients admitted to the Intensive Care Unit on returning from theatre have pulse rate and blood pressure recorded at 15 -min intervals. Temperature readings are taken 4-hourly. The urinary bladder has usually been catheterized and connected to a continuous closed drainage system utilizing a plastic bag of the Aldon type $\mathrm{C}$ variety. This permits the collection of hourly samples for measurement and urgent analysis, if necessary.

After measurement all samples are pooled and the 
24-hr volume is then submitted to the laboratory for analysis of sodium, potassium and total nitrogen content. Gastro-intestinal aspirates are also collected, together with all secretions from body cavities. Samples are taken of capillary blood on return from theatre for estimation by Astrup's method of acid-base status. Oxygen tension is determined when necessary by a Radiometer Clark electrode, but oxygen is given routinely by nasal catheter. Intravenous fluids are given according to requirements and frequent changes of position are carried out by the nursing staff. The objectives of treatment within the Unit are to prevent, as far as possible, deviations from normal in tissue metabolism. Four parameters are considered to be of importance and these receive most of the attention of the staff of the Unit.

\section{Tissue perfusion}

A satisfactory cardiac output provides the basis of good tissue perfusion. Arterial blood pressure is used as a monitor, and is measured at $15-\mathrm{min}$ intervals. Comparison is made with individual preoperative figures. A fall in systolic pressure of more than $20 \mathrm{mmHg}$ is regarded as significant. The commonest cause of reduced blood pressure is in our experience insufficient replacement of blood and fluid lost at operation. When systolic pressures are low, measurement of central venous pressure has proved of considerable help in assessment of general condition. After major cases a cannula is left in the vena cava so that central venous pressure can be measured as required. If no cannula has been left in situ an 'Intracath' catheter may be inserted into the external jugular vein comparatively easily and passed into the superior vena cava. If both arterial and venous pressures are reduced, transfusion with blood is commenced and circulating blood volume checked by isotope dilution using ${ }^{131}$ I-labelled albumin, measured by the Volumetron. Post-operative haemorrhage has not been a common occurrence, but an increase in the vascular space due to rewarming after a fall in total body temperature during operation has been encountered, and may require transfusion to correct the fall in pressure.

\section{Acid-base status}

Blood gas analysis was carried out routinely to determine $\mathrm{CO}_{2}$ tension and blood $\mathrm{pH}$. The oxygen tension in arterial blood was not always measured unless specific indications were present. Oxygen was administered by nasal catheter and in most cases this maintained a $\mathrm{Po}_{2}$ of around $100 \mathrm{mmHg}$ when check estimations were performed. Adequate oxygen tension is particularly important in Group 3 admissions to the Unit, since, as Nunn \& Payne (1962) point out, hypoxaemia is common after surgery and is accentuated by pre-existing respira- tory disease. Indeed, in the latter group physiological pulmonary shunts increase during surgery (Hobsley, 1963) and there is evidence that segmental collapse occurs during operation. Bronchoscopy may be necessary to re-aerate collapsed segments, although postural drainage and physiotherapy can usually improve ventilation.

Hydrogen ion concentration is easily measured by the Astrup capillary method (Astrup et al., 1960). Alterations of $\mathrm{PCO}_{2}$ have been common. $60 \%$ of all general surgical admissions showed elevations of $\mathrm{PCO}_{2}$ above the pre-operative level and $20 \%$ showed values of more than $60 \mathrm{mmHg}$ at some stage during the first $12 \mathrm{hr}$ after surgery. The reasons for these changes varied from an occasional incomplete reversal of relaxant and in other cases a combination of underlying chest disease, prolonged operation and incision splintage of respiratory muscles. Four cases admitted to the Unit for treatment had been returned to a general surgical ward following operation, and transfer was necessary following anoxic cardiac arrest. All cases exhibiting $\mathrm{CO}_{2}$ retention were ventilated using a Bird respirator. On some occasions, for example, a patient presenting with a ruptured aortic aneurysm and having obstructive airways disease, prophylactic assisted respiration was employed. The oxygen consumption of the respiratory muscle at rest is about $2-3 \%$ of the total oxygen consumption of the body as a whole. The proportion rises as high as $50 \%$ if the work of breathing is increased (Cherniack \& Cherniack, 1961). Dammann, Thung \& Littlefield (1963) introduced controlled respiration after surgery to reduce respiratory work, and while fully controlled respiration may not be necessary following operation, assisted respiration triggered by the patient can be beneficial in such cases. The endo-tracheal tube is left in place after surgery and removed $24 \mathrm{hr}$ later if the patient's condition warrants. If further assisted ventilation is required, tracheostomy is performed and ventilation continued via this route. Care must be given to the removal of bronchial secretions. A sterile whistle-tip catheter, size $F 8$, is connected via a ' $Y$ ' connection to the piped suction supply and passed down first the right and then the left main $N$ bronchus. A finger is placed over the open limb of the ' $Y$ ' piece and suction directed to the tip of the catheter which is withdrawn once only. Traumatic $\omega$ abrasion of the tracheal and bronchial epithelium is thereby reduced to a minimum and all secretions $\stackrel{\varrho}{\subset}$ are removed. A bacterial culture should be taken on termination of ventilation, and on withdrawal of ? the endotracheal tube from the patient.

Metabolic acidosis is occasionally encountered. Prolonged anaesthesia (Cranston, Pepper \& Ross, 1955) may produce this type of $\mathrm{pH}$ change but poor tissue perfusion is in our experience the commonest cause. Hypothermia may influence the metabolism 
of administered acid and ACD. Bank blood when transfused may, by virtue of the $54 \mathrm{mEq} / \mathrm{l}$ of additional acid (Galletti \& Brecher, 1962), help to induce a state of metabolic acidosis. It is unusual for $\mathrm{pH}$ change to persist, however, in the presence of good perfusion after vascular surgery (Johnstone, Lawson \& Mucklow, 1965). If significant hypotension occurs, as for example during cardiac arrest, profound acid base changes occur and $8.4 \%$ bicarbonate is given immediately to provide $100 \mathrm{mEq}$. Acid-base figures are determined and the calculated base deficit corrected.

Metabolic alkalosis has been encountered on several occasions. It has been demonstrated following cardio-pulmonary bypass (Gerbode, Osborn \& Johnstone, 1960; Raison, 1965). Its aetiology is uncertain, but transfusion of large volumes of ACD blood may be a factor, particularly if sodium retention is present (Litwin et al., 1958). Significant reduction in serum potassium levels has not been associated with this change, but increased urinary excretion of fixed acid has been noted (Fig. 1). $\mathrm{CO}_{2}$
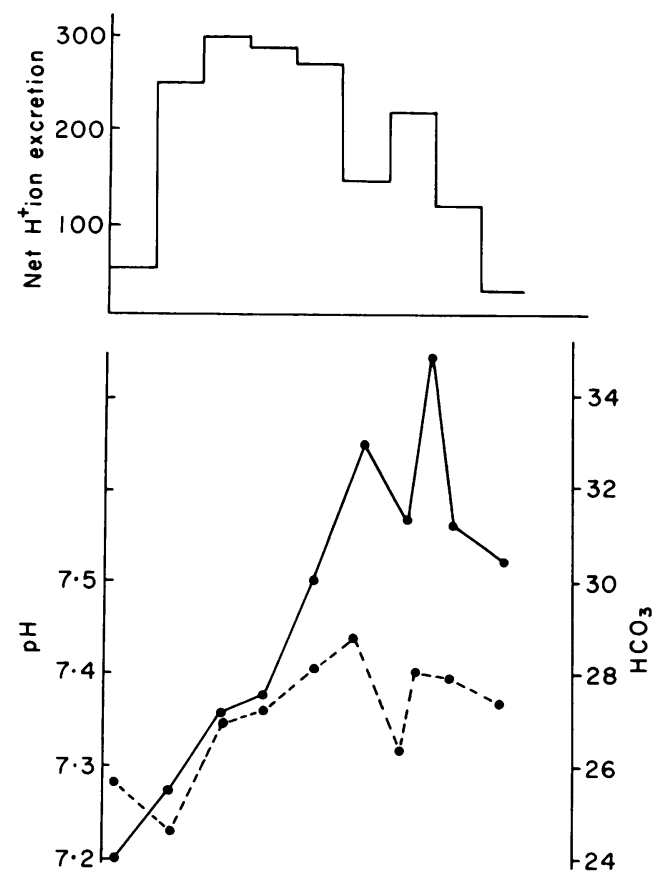

Fig. 1. Net $\mathrm{H}^{+}$ion excretion and serum $\mathrm{HCO}_{3}^{-}$concentrations during 9 days following pharyngo-laryngectomy.

tension is not obviously elevated nor is there any evidence of over-ventilation. Its affects seem to be innocuous since no adverse reactions have occurred during its presence.

\section{Renal function}

Renal function is closely observed in all cases admitted to the Unit. Major vascular cases and patients undergoing extensive surgical resection have indwelling urethral catheters of the Gibbon variety. Diuresis commences in the theatre and tissue hydration and urine flow are important factors in preventing both high and low output renal failure when unexpected complications such as hypotension occur. Paratubular perfusion or enhanced tubular urine flow rate are probably the effective means by which this is accomplished. Provided adequate fluid is administered, post-operative antidiuresis can be inhibited.

Urine volumes are measured hourly. This in itself is not always a safeguard as renal failure may be present in spite of daily urine volumes which are greater than $1000 \mathrm{ml}$. Specific gravity measurements likewise can prove misleading, particularly when Dextran is being administered in conditions where tubular reabsorption of water predominates. High urinary Dextran concentrations may then occur (Artuson, Granath \& Thoren, 1964), and urine specific gravity may rise above 1050 . Urine osmolality is helpful when urgent information is required and analysis of the 24-hr volume will give specific information about total nitrogen excretion and urinary urea so that an approximate concentration ratio for the kidney can be determined by comparing urine and serum values. Urinary flow rates of $1 \mathrm{ml} / \mathrm{min}$ are minimal requirements and if very low values occur, i.e. $10 \mathrm{ml} / \mathrm{hr}$ or less, it is our practice to give $50 \mathrm{~g}$ mannitol over a $1-2 \mathrm{hr}$ period to promote diuresis. This usually establishes the flow of urine, but if no response is obtained it is likely that oliguric renal failure has occurred. If mannitol infusions are given, increased secretion of sodium may occur, but in doses of $100 \mathrm{~g} /$ day this is not excessive and serum levels are not affected (Cheney, Rand \& Lincoln, 1964).

Intravenous fluids are supplied in an average volume of $3 \mathrm{l} /$ day to a $70-\mathrm{kg}$ adult. Five per cent fructose or dextrose are the basic infusions and electrolytes are added according to calculated deficits. In practice this amounts to supplying $80-140 \mathrm{mEq}$ sodium and $60-80 \mathrm{mEq}$ of potassium per $24 \mathrm{hr}$.

\section{Nutrition}

The nutritional state of the patients admitted to an Intensive Care Unit should be considered, although few patients admitted for major abdominal procedures or vascular surgery are malnourished and relative starvation for a few days is not harmful. When obstructive lesions of the pharynx and oesophagus are present, early introduction of parenteral nutrition is desirable. It may also be required after extensive plastic surgery to the head and neck. In the present series four patients were admitted to the Intensive Care Unit after pharyngo-laryngectomy and pharyngo-colo-gastrostomy effected by means 
of a pedicle graft. Full parenteral nutrition was supplied to these patients and each received $15 \mathrm{~g}$ of nitrogen per day in the form of $10 \%$ amino-acid hydrolysate (Aminosol) covered with 1 litre of fat emulsion (20\% Intralipid) and 1 litre $20 \%$ fructose. Care was taken to infuse carbohydrate with, or before, the amino acid solution to achieve maximal utilization, and minimum daily intakes of 2600 calories were achieved. Sodium intake was more than adequate due to the high content of this ion in the hydrolysate, and potassium supplements were supplied to cover the nitrogen administered and to replace measured losses. Full vitamin supplements were given. Overall nitrogen balance was improved in these cases and weight loss reduced to a minimum (Fig. 2). Parenteral nutritional therapy has also been

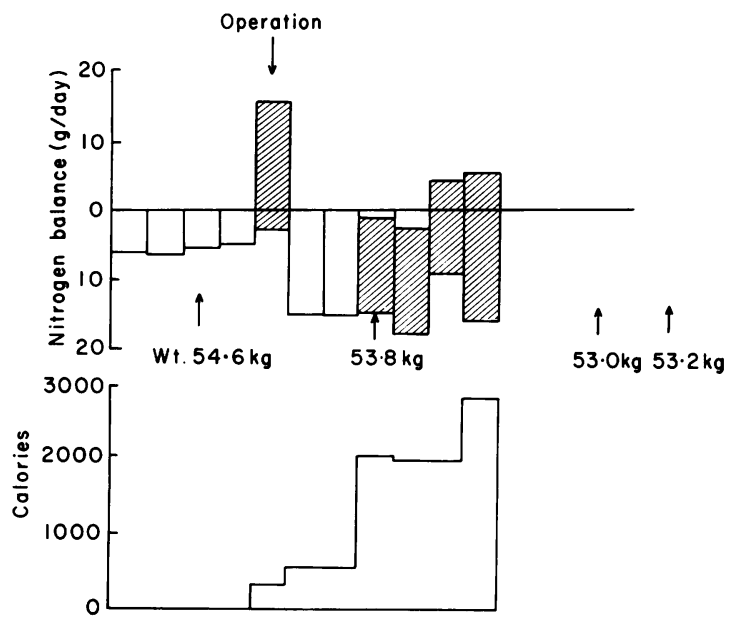

FIG. 2. Nitrogen balance and total daily calorie intake after pharyngo-laryngectomy and colon graft. $\mathrm{N}_{2}$ output plotted below the line, and $\mathrm{N}_{2}$ intake as attached area from bottom of output.

given in two cases admitted because of abdominal and thoracic trauma, requiring intensive treatment and assisted ventilation for many days. Three cases treated after emergency mesenteric vascular procedures also required prolonged therapy because of 'second look' operations and multiple resections. Variations from the basic nutritional regime have been employed for patients admitted after a portacaval shunt. In these cases amino-acid hydrolysate has been withheld to avoid administering the free ammonia which is present in the solution. Fat emulsions, although well tolerated in the cirrhotic patient (Lawson, 1965), were not used because of transient rises in SGOT which have been demonstrated previously. The basic infusion in these patients was $10 \%$ or $20 \%$ fructose which provided a minimum calorie supply of 1200 calories/day.

Close attention to the biochemical parameters outlined previously contributes to the improved

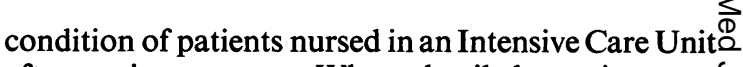
after major surgery. When detailed nursing pro- $c$ cedures and the better general nursing care that is $\overrightarrow{=}$ given to such patients is considered in addition, it is not surprising that even after extensive operations a $\frac{}{\circ}$ successful result is to be expected. Modern surgicalo practice demands a wider introduction of $\operatorname{such} \vec{\Phi}^{\circ}$ Units.

\section{Summary}

Intensive nursing care and treatment is desirable $\overrightarrow{\vec{H}}$ during the post-operative phase of some types of ${ }_{\sigma}^{\omega}$ general surgery. The indications for admission of such cases to the Intensive Care Unit are:

(1) Elective admission following surgery of and extensive or prolonged nature performed on healthy patients.

(2) Elective admission, after surgery of a lessi complicated variety than Class (1) but performed on poor risk patients.

(3) Emergency admission principally required when cardiac or respiratory insufficiency develops unexpectedly during or after surgery.

The requirements of patients admitted to $\operatorname{such}_{\vec{\varphi}}$ Units varies from close observation to full intensive treatment. Equipment for automatic monitoring and recording of vital signs is desirable but not essential, but a detailed record of all changes occurring in the patient and treatment given should be kept by the nursing staff. Facilities for collection and analysis of $\frac{0}{\varnothing}$ aspirates, drainage and urine must be available. $\stackrel{2}{\square}$ Blood gas analysis is particularly important and $\overrightarrow{\overrightarrow{0}}$ apparatus for such determinations is preferably located within the Unit.

Provision must be made for mechanical ventilation-both therapeutic and prophylactic-and the staff should be fully conversant with one principal ventilator.

Four parameters of body function are considered to be of prime importance: (1) tissue perfusion, (2) acid-base status, (3) renal function, and (4) nutrition.

The results of close attention to such factors is $\frac{7}{2}$ discussed.

\section{Acknowledgments}

I should like to express my thanks to Professor A. L. N d'Abreu for permission to study patients admitted to the $\omega$ I.T.U. in the Department of Surgery at the Queen Elizabeth Hospital.

\section{References}

Artuson, G., Granath, K. \& Thoren, L. (1964) Renal $\square$ excretion of low molecular weight dextran. Acta chir. 음 scand. 127, 543.

Astrup, P., Jorgensen, K., SiggaArd-Andersen, O. \& $\frac{\stackrel{\rho}{\Phi}}{\mathbb{D}}$ ENGEL, K. (1960) Acid base metabolism. A new approach. $\varrho$ Lancet, $\mathbf{i}, 1035$.

Cheney, F.W., RAND, P.W. \& Lincoln, J.R. (1964) Mannitolinduced diuresis. Arch. Surg. 88, 197. 
CherniaK, R.M. \& CherniAK, L. (1961) Respiration in Health and Disease, p. 30. Saunders, Philadelphia.

Cranston, W.I., PePper, M.C. \& Ross, D.N. (1955) Carbon dioxide and control of respiration during hypothermia. J. Physiol. (Lond.), 127, 380.

Dammann, J.F., Thung, N. \& Littlefield, J.B. (1963) The management of the severely ill patient after open-heart surgery. J. thorac. cardiovasc. Surg. 45, 80.

Galletti, P.M. \& Brecher, G.A. (1962) Heart Lung Bypass, p. 222. New York.

Gerbode, F., Osborn, J.J. \& Johnstone, J.B. (1960) Experience with perfusion hypothermia using an improved rotating disc oxygenator. Thorax, $15,185$.

HoBsley, M. (1963) Respiratory disturbances caused by general surgical operations. Ann. roy. Coll. Surg. Engl. 33, 105.

Johnstone, J.H., Lawson, L.J. \& Mucklow, R.G. (1965) Metabolic changes after aorto-iliac occlusion. Brit. med.J. ii, 974.

LAwson, L.J. (1965) Parenteral nutrition in surgery. Brit. J. Surg. 52, 795.

Litwin, M.S. (1958) Extracorporeal Circulation, p. 350. Allen.

NunN, J.F. \& PAYNe, J.P. (1962) Hypoxaemia after general anaesthesia. Lancet, ii, 631.

RAISON, J.C. (1965) Acid base changes and tissue respiration in extracorporeal circulation. Ann. roy. Coll. Surg. Engl. 37, 93.

\section{Discussion to the paper by L. J. Lawson}

Brooks. Studies such as those carried out by Dr Lawson are long overdue in this country. There are, however, aspects upon which I would like to comment.

In the field of acid-base balance a number of anomalies may be present. For example, porta-caval anastomosis may produce a number of metabolic disorders which include hyponatraemia, hyper- or hypo-kalaemia, intolerance to a high oral protein intake which precipitates hepatic coma even in the absence of pre-operative hepatic dysfunction, and metabolic acidosis (Hubbard, 1958). The fall in bicarbonate ion concentration in most cases of porta-caval anastomosis is of much greater significance because patients with cirrhosis of the liver usually have a raised plasma bicarbonate concentration. They also have low $\mathrm{PCO}_{2}$ because of increased alveolar ventilation, and the blood pH is therefore high (Brooks, 1967). When liver damage is very severe, e.g. if liver necrosis develops, a marked metabolic acidosis is observed which does not respond to infusion of sodium bicarbonate.

The metabolic changes that occurred during replacement of the abdominal aorta as shown on Dr Lawson's slide, are very similar to those found in that type of case by myself. It is quite true that if the aorta is clamped for only half an hour during an elective procedure, for example for claudication where anastomotic vessels have already developed only a moderate degree of metabolic acidosis is found together with a small rise in blood lactate and blood pyruvate.

If, however, the aorta is replaced during an emergency operation for a leaking abdominal aneurysm and the aorta is clamped for long periods, e.g. $3 \mathrm{hr}$ or more, then a marked metabolic acidosis develops and this is associated with hypotension that does not respond to blood transfusion (Brooks \& Feldman, 1962; Knight, 1963). Correction of the metabolic acidosis by infusion of sodium bicarbonate solution results in a dramatic rise in blood pressure in a similar manner to that reported yesterday (McConn, Fig. A8, p. 224).

Post-operatively a metabolic alkalosis frequently develops and this is thought by Moore (1959) to be due to the citrate infused with stored blood. However, an increase in the plasma bicarbonate ion concentration can also be observed following blood transfusion. It is also of interest to note that following major vascular and cardiac surgery the blood lactate and pyruvate may be raised even in the presence of a metabolic alkalosis.

Acute renal failure is a relatively rare condition but post-operative oliguria is commonly seen. Oliguria very rarely develops into acute renal failure and I am not convinced that mannitol is at all effective in preventing the onset of acute renal failure. Sometimes mannitol will produce a diuresis for very brief periods before acute renal failure is fully developed and is then later completely ineffective. The mechanism of this is too involved to discuss at this moment but it is possible that in these circumstances mannitol is filtered through glomeruli that lie near the surface of the kidney from which loops of Henle arise which are confined to the cortex, never entering the medulla and therefore the concentrating areas.

It is correct to stress the importance of parenteral nutrition in some cases. We too have been able to obtain a positive nitrogen balance in post-operative patients by this means, using a synthetic amino acid preparation containing sorbitol.

\section{References}

Brooks, D.K. (1967) Resuscitation, p. 57. Edward Arnold, London.

Brooks, D.K. \& Feldman, S.A. (1962) Metabolic acidosisA new approach to neostigmine-resistant curarisation. Anaesthesia, 17, 161 .

HubBaRD, T.B. (1958) Carcinoma of the head of the pancreas: resection of the portal vein and portacaval shunt. Ann. Surg. 147, 935.

KNIGHT, P.F. (1963) Anaesthesia for the leaking abdominal aneursym. Anaesthesia, 18, 151.

MOORE, F.D. (1959) Metabolic Care of the Surgical Patient, p. 858. Saunders, Philadelphia. 